\title{
Pricing Strategy of China's Ride-haling Platforms in Two-sided Market
}

\author{
Yiming Chen ${ }^{1}$
}

\author{
${ }^{1}$ Huazhong University of Science and Technology, School of Foreign Language, Wuhan, China, 430000 \\ Corresponding author. Email: 1149580128@qq.com
}

\begin{abstract}
Two-sided markets focus on two-sided consumers' economic behavior and platforms that provide services for both consumers and sellers. This paper illustrates the basic definition and market structures of the two-sided market, combining with hotspot problems of online ride-hailing platforms in China related to the two-sided market. Referring to the basic pricing strategy of the two-sided market, research on pricing strategies of ride-hailing platforms is made to analyze the pricing and profitability of the platforms.
\end{abstract}

Keywords: Pricing Strategy, Two-sided Market, Ride-hailing Platform, China, Platform competition

\section{INTRODUCTION}

With the evolution of media technology and mobile network technology, online ride-hailing, shared bicycles, and shared cars have become an indispensable part of people's lives in China. With the application of big data technology, the rise of online ride-hailing platforms has alleviated the long-standing problem of getting a taxi in China's urban areas. On the one hand, ride-hailing platforms solve the information asymmetry of the taxi industry by providing personalized commuting demands of consumers and effectively reduce the service cost of drivers. On the other hand, as the market share grows wildly, problems of ride-hailing platforms such as burnt subsidies, free fare increases, pricing opaque have emerged, and major platforms are at a loss. Therefore, reasonable pricing strategies are in urgent need to establish to promote the sustainable development of online ride-hailing platforms.

The online ride-hailing platform serves as an intermediary to provide services for both consumers and drivers, which constitutes a two-sided market. Referring to the basic pricing strategy of a two-sided market, researches on pricing strategies of ride-hailing platforms is made to analyze the pricing and profitability of the platforms.

\section{BACKGROUNDS INFORMATION ON RIDE-HALING MARKET IN CHINA}

With the development of Internet technology and the popularization of smartphones, the commuting industry has built a close connection with mobile networking. In 2010, Uber born as the world's first ride-hailing platform, then a large number of ride-hailing platforms such as Lyft, Hailo and Zabcab have emerged in Europe and the United States. As a matter of fact, Uber operates in more than 400 cities in more than 70 countries worldwide, which shows the potential of the ride-hailing market. In March 2012, as the first ride-hailing platform, YaoYao Taxi, launched its service in China, many ride-hailing platforms like DiDi Taxi, KuaiDi Taxi, YiDao Taxi have made their way to occupy the market for convenient, diversified and personalized services. The prevalence of online ridehailing platforms has led to the rapid growth in the scale of consumers and transactions on the online ride-hailing platform. However, to occupy the whole market share, online ride-hailing platforms have provided large-scale subsidies to consumers and drivers, creating vicious competition. Until August 1, 2016, DiDi Taxi's acquisition of Uber China marked the end of the price competition in the ride-hailing industry.

However, the pricing problem of the platforms is still particularly prominent after the competing period. Firstly, it is the issue of subsidies for online ride-hailing platforms. The subsidy strategy has achieved rapid user growth in a short period, which is conducive to the rapid increase of the platform's market share, but long-term 
subsidies will lead to a loss. Therefore, after stopping large-scale subsidies, how the platform should maintain and further expand the scale of consumers and drivers, and improve user loyalty and satisfaction, is a practical problem that needs to be faced and resolved. Secondly, the pricing strategy of online ride-hailing platforms is not transparent. Different from traditional taxi services, online ride-hailing platforms implement variable pricing methods. By setting up a mark-up function within the system, according to vehicle resources and the actual needs of consumers, it uses price levers to adjust commuting fees during peak hours or holidays and other special car usage periods. Nevertheless, this pricing method has the drawbacks of the arbitrary price increase and unexpected charges due to the opaque pricing process in practice, which harms consumers' rights and interests. The third aspect is the effective competition and profitability of online ride-hailing platforms. Currently, there are not many platform companies that are actually profitable in the market. Not relying on price competition to achieve effective competition and thereby increase profitability is a question that platform companies must seriously consider.

Besides, the online ride-hailing platforms set prices for both consumers and drivers simultaneously, which virtually increases the risk of platform pricing. Because the level of pricing for users on one side will indirectly affect the scale of users on the other side, it affects the scale of users on the entire platform. For example, reducing subsidies for consumers and increasing platform usage fees will reduce the number of consumers and reduce the attractiveness of the platform to drivers. The number of consumers and drivers will likely decrease at the same time. These problems will seriously affect online ride-hailing platform companies' development and are also not conducive to effective competition in the online ride-hailing market. Therefore, a reasonable pricing strategy will need to be explored to promote the online ride-hailing market's sustainable development.

\section{TWO-SIDED MARKET}

Caillaud and Jullien (2003) proposed the two-sided market by analyzing the price competition model of intermediary service providers in the bilateral market and proposed the characteristics of bilateral market network externalities and price discrimination[1]. In the same year, Rochet and Tirole (2003) defined a two-sided market platform as a platform that attracts two-sided users through a reasonable price strategy to generate revenue[2]. Armstrong (2005) defined the platform in a two-sided market as a user's transaction intermediary, and the user's utility on one side is compared with the network externalities of the other side[3]. Rochet and Tirole (2005) further suggested that the total amount of fees charged by the platform to users on both sides of the bilateral market is constant, and the transaction volume of the platform changes when the prices of both users are changed[4]. In terms of the features of the two-sided market, Rochet and Tirol (2003) proposed that the twosided market has two features of network externality and non-neutral price structure[2]. Regibeau (2005) further pointed out that the two-sided market has three typical features as market structure, cross-network externality and price structure that are not neutral[5]. Holland (2007) takes the maximization of platform revenue as the starting point and proposes two features of the two-sided markets: one is that the platform attracts users from both sides and benefits from them by reducing transaction costs; The second is the complementary needs that the number of users on one side can affect the utility of users on the other side[6].

Armstrong (2006) summarized the pricing factors in two-sided markets as cross-network externalities, platform charging methods, and user ownership structure[3]. Che (2010) divides the participants in the two-sided market and summarizes the factors affecting pricing from the users, platforms, and service providers[7]. Specifically, the factors that affect user pricing include cross-networking among users, externalities, user demand price elasticity and user attribution conditions. Factors affecting platform pricing include platform differentiation and competition. Factors affecting service provider pricing include service provider market power.

\section{RIDE-HAILING PLATFORM}

\subsection{Information Transparency}

The information transparency of ride-hailing platforms relies on big data technology and can efficiently collect, calculate, and disclose data related to the operation process. In the process of booking a vehicle, consumers and drivers can consider each other's basic personal information, geographic location, and waiting time, as well as grasp the platform's real-time freight rate and traffic congestion. Therefore, the platform's information transparency is relatively high, which significantly reduces the information asymmetry between users, reduces user costs, and improves the operational efficiency of the entire travel market.

\subsection{Declining Cost}

Ride-hailing platform companies are different from the traditional manufacturing industry. With the expansion of production scale in traditional enterprises, the traditional industry's cost continues to decrease. After reaching a critical scale, the production of unit products increases, and the industry's cost is on the rise. When the ride-hailing platform develops a ride-hailing app, it needs to invest relatively high costs. After the software is put into use, the cost of maintenance keeps low, and the 
marginal cost is close to zero. Therefore, after the online car-hailing platform reaches a particular scale, the service cost of the platform will gradually decrease.

\subsection{Full Competition}

Compared with the traditional taxi market, online ride-hailing arises from the massive demand in the commuting market and is relatively less subject to government intervention in terms of price and quantity. Therefore, the competition in the online car-hailing market is relatively sufficient. The information on the online ride-hailing market is highly transparent. Users can obtain information faster and consumers can choose low-priced, high-quality services in a short time, and drivers can choose orders with high profits and close distances. Secondly, registration cost and switching cost of the platform for consumers and drivers are lower. For most online ride-hailing platforms, users only need to register to access the platform, and there is no long-term contract constraint between the platform and users. Users can switch between different ride-hailing platforms at lower or no cost. Thirdly, the online ride-hailing platform relies on Internet technology and has the Matthew Effect.

\section{PRICING STRATEGY}

In a competitive market, competition among platforms is fierce, and their pricing is also affected by many factors. Based on the research mentioned above on the pricing of online ride-hailing platforms, relevant strategic recommendations are put forward for the pricing of online ride-hailing platforms. The pricing of ridehailing platforms is affected by factors such as crossnetwork externalities between users, user expectations, and the efficiency of platform technology matching. The action is consistent with the pricing of monopolistic ridehailing platforms. Therefore, online ride-hailing platforms can adopt the following pricing strategies.

\subsection{Preferential Pricing}

Considering the cross-network externalities between consumers and drivers, platforms may adopt the preferential pricing strategy to set low prices for users of the cross-network with strong externalities and high price for users with weak externalities. Hagiu (2004) constructed a model in which the seller enters the twosided market before the consumers to ensure that pricing affects two-sided market[8]. Setting a low price for active users can increase the frequency of use and increase the number of transactions on the platform. Low prices can expand the scale of users and increase user stickiness.

\subsection{Technical Improvement}

Improve the efficiency of technology matching, reduce the pricing level of different users, and improve the efficiency of order matching with higher platform competitiveness, which can quickly realize the matching between consumers and drivers, improve user experience, and increase the number of orders and the scale of transactions. By improving the efficiency of technology matching and lowering users' price, it can attract users to access the platform and enhance the level of user utility.

\subsection{Differentiated Services}

The pricing of ride-hailing platforms for users is positively related to the level of differentiation of the products and services provided by the platform. The higher the level of platform differentiation, the stronger the pricing power. Besides, differentiated operations can increase user loyalty and enhance the market power of the platform, which is the primary way for the platform to obtain profit. Therefore, major online ride-hailing platforms should improve product categories, give play to their comparative advantages, and conduct differentiated operations. The platform's differentiated business strategy can be specifically considered from the following aspects:

- Service differentiation. Platform services are ultimately oriented to both consumers and drivers, and user needs must be placed at the core. Platforms should consider more on the needs of consumers, improve platform management, improve service levels, and at the same time improve the service to drivers, meet the demands of drivers.

- New products and services. The platform should speed up the development of new products and continue to extend the business area. The platform can also develop new products and services based on the commuting needs of a particular group. For example, Uber extends its services to shopping, dining, medical care, entertainment and other fields to fully meet users' needs.

- Strengthening cooperation and provide value-added services. Strengthening collaboration with thirdparty payment platforms and map providers, improving the payment system and information matching functions of the platform, ride-hailing platforms can improve user experience, introduce advertisers, and provide consumers with integrated services such as accommodation, dining, and tourism.

\subsection{Single-homing Consumers}

In the online ride-hailing market, the multi-homing consumers and drivers will reduce the pricing ability of platforms. In order to obtain a better user experience or enjoy subsidies from different platforms, both consumers and drivers have the motive of multi-attribution. This leads to the potential risk of losing users on the online ride-hailing platforms, which will bring losses to the 
platforms. Therefore, in order to improve the pricing power of the platform and increase the attractiveness of the platform to users, innovative services must be used to increase the proportion of single home users and increase the stickiness of users. For consumers, it is possible to cultivate consumer habits through a membership system, recharge activities, cash discounts, or provide highquality services to increase consumer stickiness, so that consumer users are willing only to access the platform. For drivers, platforms can sign exclusive contracts with drivers or train their own drivers, or the platform can establish long-term cooperation with drivers to provide drivers with more favorable conditions for cooperation compared to other platforms, forming a single-homing expectation for drivers users. By improving the singularity of users and enhancing the stickiness of the platform, it will increase the platforms' pricing power in the two-sided market and increase the profitability of the platforms.

\section{CONCLUSION}

In any two-sided market, pricing strategies are the focus of attention. The online ride-hailing platform is a typical two-sided market connected to consumers with travel needs and drivers who provide travel services on the other. The needs of consumers and drivers complement each other, and the quantity on one side is positively related to the utility of the other side, which has a significant cross-network externality between the two sides. The overall pricing level and pricing structure of consumers and drivers will directly affect platform transactions. The pricing of the platform must take into account the attributes of the bilateral market. This paper combines the basic pricing theory of the two-sided market with the features of online ride-hailing platforms and derives the factors affecting platform pricing and profits. Ride-hailing platforms should provide preferential pricing based on the cross-network externalities between consumers and drivers, provide differentiated products based on the price elasticity of consumers and drivers' demand, lower the price of active users on the platform, improve the efficiency of matching, and reduce User pricing improves the competitiveness of the platform. Therefore, online ridehailing platforms can carry out differentiated operations to improve the pricing strategy by increasing the singular type of user.

\section{ACKNOWLEDGMENTS}

I am overwhelmed in all humbleness and gratefulness to acknowledge my depth to all those who have helped me to put these ideas, well above the level of simplicity and into something concrete.

\section{REFERENCES}

[1] Caillaud, B., \& Jullien, B. (2003). Chicken \& egg: Competition among intermediation service providers. RAND journal of Economics, 309-328.

[2] Rochet, J. C., \& Tirole, J. (2003). Platform competition in two-sided markets. Journal of the european economic association, 1(4), 990-1029.

[3] Armstrong, M. (2006). Competition in two-sided markets. The Rand Journal of Economics, 37(3), 668-691.

[4] Rochet, J. C., \& Tirole, J. (2005). Two-Sided Markets: A Progress Report, erscheint in. Rand Journal of Economics.

[5] Regibeau, P. (2005). A comment on Evans, Hagiu and Schmalensee. CESifo Economic Studies, 51(2/3), 225.

[6] Holland, M. (2007). Two-sided markets: A challenge to competition policy. The First Annual Competition Commission.

[7] Che, W. (2010). Research on the Pricing Model and Profit Influencing Factors of E-commerce C2C Platform (Doctoral dissertation).

[8] Hagiu, A. (2004). Optimal pricing and commitment in two-sided markets. The Rand Journal of Economics, 20, 658-670. 